\title{
Accesibilidad web para los discapacitados: ¿una nueva herramienta para la integración social o un nuevo motivo de exclusión social?
}

\author{
Web accessibility for the handicapped: \\ a new tool for social integration or a new reason for social exclusion?
}

\author{
Esmeralda SERRANo MASCARAQUE \\ Facultad de Documentación de la Universidad de Alcalá. C/ San Cirilo, s/n. \\ 28801 Alcalá de Henares (Madrid), esmeralda.serrano@uah.es
}

\begin{abstract}
Resumen
Actualmente, las tecnologías de la información y la comunicación (TIC), y particularmente Internet, se han convertido en el mayor canal de comunicación a nivel mundial, permitiendo desde cualquier punto y en cualquier momento el acceso a la información y al conocimiento. Esta popularización ha sido factible, en parte, porque en los últimos años los servicios administrativos e informativos basados en la Web han proliferado y, también, al aumento exponencial del número de usuarios con acceso a Internet. Pero, el hecho de que la sociedad de la información y las TIC supongan una oportunidad sin precedente para favorecer la integración social y laboral de las personas con discapacidad no descarta el riesgo inasumible de que la falta de acceso a las mismas por parte de este colectivo acabe convirtiéndose en una nueva barrera para el mundo de la discapacidad. Se realiza un acercamiento al mundo de la discapacidad (legislación, directrices, tipos de deficiencias, principales problemas de integración...) y se detectan los principales problemas que dificultan su acceso a Internet, así como las pautas de accesibilidad imperantes y los instrumentos que contribuyan a mitigar las barreras que dificultan el acceso a la información en la Red.
\end{abstract}

Palabras clave: Accesibilidad Web. Invidentes. Discapacitados. Acceso a Internet. Tecnologías de la Información y la Comunicación (TIC). Integración social. Integración laboral.

\section{Introducción}

Se estima que en el mundo hay más de 650 millones de personas con discapacidad; es decir, aproximadamente, el $10 \%$ de la población mundial. Según datos procedentes de la Comisión Europea, alrededor de 37 millones de ciudadanos europeos padecen algún tipo de discapacidad, lo que representa, también, el $10 \%$ de la población total de la Unión.

En España, de acuerdo con los resultados que arroja la Encuesta sobre Discapacidades, Defi-

\begin{abstract}
Nowadays, information and communication technologies (ICT), in special the Internet, have become the main channel of communication in the world, allowing the access to information and knowledge from every point and at every moment. This popularity has been feasible, in part, because in the last few years the administrative and informative services based on the web have proliferated, and also, because of the improvement of exponential number of users with access to the Internet. However, the growth of the society of information and ICT means an opportunity to improve the social and labour integration for disabled people, but it doesn't eliminate risks for them and maybe it will become a new barrier, an other impossibility for handicapped people. This paper examines how this fate can be avoided. It iintroduces the main aspects of disabled persons protection (legislation, guidelines, the disabled types, the main integration problems...), and, from them, the main problems of their access to the Internet are faced. The level of compliance of the accessibility guidelines is examined, and the available and possible tools to reduce access barriers are discussed.
\end{abstract}

Keywords: Web accessibility. Blind. Disabled people. Internet access. Information and Comunication Technologies (ICT). Social integration. Labour integration.

ciencias y Estado de Salud realizada por el INE (Instituto Nacional de Estadística), el porcentaje de ciudadanos que padecen algún tipo de discapacidad se sitúa en torno al $9 \%(3.528 .221$ personas discapacitadas); de éstos el 10\% corresponde a discapacitados visuales. La prevalencia de las situaciones de discapacidad está claramente relacionada con la edad. Como norma general, la probabilidad de tener una discapacidad aumenta con la edad de manera continua, aunque a partir de los 50 años este incremento se acelera notablemente. 
Además, el número de personas con discapacidad se incrementa conforme crece la población en el mundo, y se trata de un fenómeno que no afecta por igual a todos los países, sino que más de dos tercios de la cifra total de personas con discapacidad habitan en países en desarrollo (en algunos de los cuales, hasta el $20 \%$ de su población está afectada por algún tipo de discapacidad). A este dato alarmante se añade la circunstancia de que las personas con discapacidad conforman un colectivo heterogéneo, en el que cada una debe enfrentarse a barreras diferentes que han de superar de muy diferentes maneras.

Tanto las causas de las que puede derivar esa situación de discapacidad, como las consecuencias que ésta conlleva, presentan toda una serie de elementos diferenciadores que dependen de las diversas coyunturas socioeconómicas circundantes y de la actividad desplegada por los correspondientes Estados en favor del bienestar de sus ciudadanos. En este sentido, las condiciones de vida que han acompañado, tradicionalmente, a las personas con discapacidad se han visto influidas en muchos casos por determinados factores sociales (como el desconocimiento o el desamparo) que han provocado el aislamiento de este colectivo y un considerable atraso en su desarrollo. De ahí deriva el tradicional déficit de ocupación laboral que mantiene, y aún profundiza, la situación de discriminación y de falta de oportunidades para realizar un estilo de vida normalizado y autosuficiente que padecen las personas con discapacidad, obligadas a desenvolverse en una sociedad rígidamente estructurada a partir de la perspectiva del individuo "medio".

La respuesta política que recibe, en la actualidad, el fenómeno de la discapacidad es fruto de un proceso evolutivo experimentado a lo largo de los dos últimos siglos y que refleja, en gran medida, las condiciones generales de vida y las estrategias sociales y económicas propias de las diferentes épocas y áreas geográficas. La atención a las personas con discapacidad ha pasado de procurarles los cuidados básicos, a proporcionarles ciertos niveles de educación y rehabilitación que les han permitido convertirse en sujetos activos.

En la segunda mitad del siglo $X X$, la apreciación real de las aptitudes y capacidades de las personas con discapacidad experimentó un notable avance, originando una nueva concepción de la discapacidad, entendida como la interacción entre las características de una persona y su entorno; es decir, el conjunto de desventajas a las que debe enfrentarse un sujeto, como consecuencia de sus déficits personales, y de los obstáculos y condicionantes impuestos por la sociedad, que le impiden participar de forma plena y en igualdad de condiciones en el devenir social y económico de la comunidad a la que pertenece. Por tanto, la responsabilidad de atenuar y minimizar la discapacidad recae en ambos elementos de esta relación: la persona con discapacidad y la estructura social a la que pertenece.

En los últimos años, la tendencia que está inspirando los enfoques asumidos por las políticas desarrolladas en materia de discapacidad parte de una corriente que toma como base el pleno reconocimiento de los derechos civiles, políticos, económicos, sociales y culturales a las personas con discapacidad, en plano de igualdad con el resto de la población, dado que éstas no conforman un grupo social aparte, sino un colectivo más que quiere y debe participar en la vida comunitaria. Ello obliga a incluir el fenómeno de la discapacidad tanto en los debates políticos relevantes, como en los acuerdos sociales y económicos; a reconocer a las personas con discapacidad el mismo derecho que asiste al resto de los individuos de optar a las oportunidades que la vida común ofrece; a favorecer su integración a través de la educación y la formación; a valorar convenientemente las diversas barreras que, en cualquiera de sus clases, sostienen y perpetúan el problema de la exclusión que presenta este colectivo.

Existe, además, otro obstáculo que entorpece el camino hacia la consideración de estas personas con discapacidad como individuos plenamente iguales al resto de los miembros de la sociedad, y que aún debe ser superado: la denominada "invisibilidad". Se trata de un fenómeno relacionado, no tanto con esos derechos fundamentales, propiamente dichos, sino con el modo en que aquéllos les son aplicados a las personas con discapacidad, e incluso con su falta de aplicación a estas personas.

La percepción de la discapacidad como motivo de exclusión ha constituido la base para que el problema de la invisibilidad de las personas con discapacidad haya arraigado como si de un hecho natural se tratase y al que, por tanto, no cabe oponer ningún tipo de alegación; una actitud que se mantiene hasta nuestros días, ya sea de forma absoluta o de forma relativa, provocando una contradicción entre el sistema de derechos y libertades fundamentales que nuestra sociedad profesa, universalmente reconocido a todos los seres humanos, y su incorrecta aplicación, con mucho menos rigor, en el supuesto de las personas con discapacidad. 
El primer paso en la consecución de la visibilidad de las personas con discapacidad se dio cuando éstas empezaron a verse a sí mismas como sujetos (no como objetos), y enmarcaron la discapacidad entre las principales cuestiones de los derechos humanos. La estrategia para atajar el problema de la invisibilidad reside, por tanto, en la insistencia para lograr el efectivo reconocimiento de sus derechos; esto es, en la aplicación igualitaria de la totalidad de los derechos humanos en el contexto de la discapacidad. Sin embargo, todavía hoy, las personas que padecen algún tipo de discapacidad se encuentran sometidas, de manera habitual, a toda una serie de impedimentos que dificultan sus posibilidades de acceso tanto a medios de transporte, edificios e instalaciones, como a actividades de educación o formación, empleos retribuidos, etc. Concretamente, las dificultades de acceso a la educación y a la formación profesional (junto a otros factores como la ocasional falta de ayudas, las inadecuadas políticas públicas o las carencias legislativas) han sido la causa indirecta de situaciones de desigualdad en la incorporación al mundo laboral de este colectivo, cuya tasa de desempleo supera ampliamente a la del resto de personas.

Efectivamente, la mayor parte de los integrantes del colectivo de personas con discapacidad pertenece también al colectivo de personas en edad de trabajar, a pesar de lo cual la tasa de ocupación en su caso es significativamente menor que la de las personas que no se ven afectadas por discapacidad alguna. Por supuesto, los diversos tipos de discapacidad padecidos influyen de forma decisiva en las posibilidades de acceso al mercado laboral y en los índices de inserción en el mismo; así, las discapacidades visuales y auditivas son las que mayor tasa de actividad presentan. Influye también, como es lógico, el grado de preparación de la persona con discapacidad, que opera en igual sentido que para el resto de la población, por lo que a mayor nivel de estudios, más alta es la tasa de ocupación.

Esta circunstancia de relegación de las personas con discapacidad del mundo laboral se opone frontalmente a los postulados de la tendencia dominante, según la cual la diversidad representa un puntal de la sociedad moderna, en cuanto generadora de una mayor productividad para la economía; por esta razón, dejar fuera del mercado de trabajo a este grupo humano implica renunciar a un potencial de increíbles dimensiones. De hecho, la aceptación de este argumento en los sistemas caracterizados por la observancia de los principios de igualdad y justicia está favoreciendo una evolu- ción en el campo político (nacional e internacional) que contempla, como la mejor arma contra la discriminación y la exclusión social de las personas con discapacidad, la vía de la inserción laboral de este colectivo, recibiendo como contrapartida todo el potencial que estas personas pueden aportar para contribuir al desarrollo económico y social de su comunidad.

El reconocimiento de los derechos de las personas con discapacidad se ha desarrollado, principalmente, a lo largo de un lapso que abarca los tres últimos decenios, y que ha sido auspiciado, en su mayor parte, por instituciones de ámbito internacional, tales como la Organización de Naciones Unidas (ONU) y sus organismos especializados (sobre todo, destacan la Organización Internacional del Trabajo, la Organización Mundial de la Salud, la Organización de las Naciones Unidas para la Educación, la Ciencia y la Cultura y el Fondo de las Naciones Unidas para la Infancia), la Unión Europea, la Organización de Estados Americanos, la Organización de la Unidad Africana y otras organizaciones pertenecientes a diversas áreas geográficas.

Las citadas instituciones han impulsado las actuaciones necesarias para infundir en los diferentes Gobiernos la vocación de adoptar las políticas más convenientes en materia de discapacidad, así como los instrumentos precisos para dotar de efectividad y de un marco normativo a dicho reconocimiento. Se trata de directrices que, en unos casos, implican un compromiso moral y político que asumen los Estados en cuanto al desarrollo de medidas para la integración e igualdad de derechos de las personas con discapacidad, y en otros, se revisten de un carácter vinculante que obliga al establecimiento de mecanismos para hacer efectivo el cumplimiento de esos objetivos de integración.

\section{Acceso a la Sociedad de la Información y del Conocimiento}

La Sociedad de la Información está experimentando un avance tecnológico que la está conduciendo hacia la globalización; pero ésta no se hará realidad sin la mitigación total y absoluta de todas las barreras físicas y tecnológicas que dificultan el acceso básico de todos los individuos, incluso los que presenten alguna discapacidad, a esa tecnología.

Para las personas con discapacidades la evolución que las Tecnologías de la Información y la Comunicación (TIC) han experimentado repercute en su integración y participación en todos los ámbitos, especialmente el socio-económico y laboral. De ahí, la necesidad de lograr la "eaccesibilidad", que conlleva no sólo que las TIC 
sean accesibles para todos, sino también que esas TIC se diseñen de manera que se adapten a necesidades y capacidades especiales, según determinadas normas de accesibilidad, tanto para contenidos-servicios como para herramientas-dispositivos. De lo contrario, esta sociedad de la información electrónica será una nueva y progresiva barrera discriminatoria para el discapacitado; mientras que la e-accesibilidad para todos puede favorecer especialmente la autonomía del colectivo de discapacitados, convirtiendo Internet en un instrumento habilitante insustituible.

En contrapartida, hay que tener en cuenta que, globalmente considerados, este colectivo, como potencial usuario de la Red y sus servicios, también aportaría cuantiosos beneficios. Efectivamente, desde comienzos de la década de los años 90, la globalización y la revolución tecnológica están transformando la economía mundial y la vida cotidiana en todas las áreas del planeta. El primero de estos dos factores, la globalización, provoca la interdependencia de las economías en todo el mundo, originando una "economía global". En cuanto a la revolución tecnológica, ha supuesto la aparición de Internet y de nuevas Tecnologías de la Información y la Comunicación. Ante esta nueva situación, las instituciones eurocomunitarias se dieron cuenta de que era preciso modernizar en profundidad la economía para mantener su productividad frente a los grandes agentes económicos a nivel mundial y comenzaron a adoptar y ejecutar decisiones al respecto.

A priori la utilización de medios informáticos abre un acceso universal e ilimitado a las autopistas de la información; pero también provoca la llamada "brecha digital". Esta ventaja se puede convertir en un factor de exclusión y discriminación social para el colectivo que nos ocupa, entre otros.

Esta nueva era digital propugna de entrada y por imposición legal (1), la obligación de hacer accesible toda la información contenida en las páginas Web pertenecientes a organismos oficiales y aquéllas cuyo diseño o mantenimiento sean financiados con fondos públicos. Pero esta responsabilidad, y obligación de difusión de la información electrónica, lleva consigo el desarrollo de una infraestructura tecnológica adecuada. Esa base tecnológica varía en función de cada país (yendo en consonancia con el desarrollo de la propia nación). Esta desigualdad ha condicionado los principales objetivos de las políticas democráticas referidas al acceso a la información universal, abierta y libre.
De acuerdo con esta línea de acción, la UE ha apostado de un modo decisivo, como no podía ser de otra forma, por aprovechar el potencial de la sociedad basada en el conocimiento y de las nuevas Tecnologías de la Información y la Comunicación (TIC) para conseguir que nadie quede excluido y atender, especialmente, las necesidades de las personas con discapacidad y su acceso al empleo. Por su parte, España cuenta con un considerable número de normas (La Constitución, artículos 9, 10, 14 y 49; la Ley 13/1982, de 7 de abril, de Integración Social de los Minusválidos; la Ley 51/2003, de 2 de diciembre, de Igualdad de Oportunidades, no Discriminación y Accesibilidad Universal de las Personas con Discapacidad, etc.) que han dotado al ordenamiento jurídico español de las bases legales necesarias para regular la igualdad de oportunidades.

La regulación sobre "e-accesibilidad" procede del legislativo estatal, que ha dictado, hasta la fecha, las leyes relativas a telecomunicaciones, servicios de la sociedad de la información y firma electrónica (Ley 59/2003, de 19 de diciembre, de Firma Electrónica y Ley 11/2007, de 22 de junio, de Acceso Electrónico de los Ciudadanos a los Servicios Públicos); aunque las disposiciones referidas al acceso de las personas con discapacidad a las TIC se encuentran también incluidas en otras normas relacionadas con la materia (Ley 34/2002, de 11 de julio, de Servicios de la Sociedad de la Información y de Comercio Electrónico (LSSICE) y Ley 51/2003, ya mencionada anteriormente). Sin embargo, aún se aprecia en España (2) una escasez de legislación que obligue a eliminar las barreras de los productos y servicios relacionados con la comunicación, la información y la señalización; concretamente, respecto de los aspectos técnicos de Internet, existen problemas de accesibilidad en los que se está trabajando, fundamentalmente a partir de la transposición de la Directiva sobre el Comercio Electrónico por la Ley 34/2002, de 11 de julio, de Servicios de la Sociedad de la Información y de Comercio Electrónico; pero la constante aparición de nuevos servicios y tecnologías hace imposible eliminar todas las barreras (en servicios como la teleformación, la banca en línea, la Administración en línea, la Sanidad en la red, los servicios de compra a través de la red, etc.). Hemos de señalar, como un gran avance significativo, la reciente aprobación del Real Decreto 1494/2007, de 12 de noviembre, por el que se aprueba el Reglamento sobre las condiciones básicas para el acceso de las personas con discapacidad a las tecnologías, productos y servicios relacionados con la sociedad de la información y medios de comunicación social. Este Real Decreto es- 
tablece la obligatoriedad de no sólo hacer accesibles las páginas Web pertenecientes a organismos públicos, sino que éstas deberán cumplir como mínimo los niveles 1 y 2 de accesibilidad recogidos en la Norma UNE 139803:2004.

\subsection{Incorporación de España a la Sociedad de la Información: Internet}

Según el Informe del Panel de Hogares de la XV Oleada del Observatorio de las Telecomunicaciones y la Sociedad de la Información (2007), elaborado por Red.es, entidad del Ministerio de Industria, Turismo y Comercio, el número de internautas españoles, mayores de 15 años, asciende a cerca de 20 millones (3) (19.690.000), lo que constituye un $52,4 \%$; de los cuales, el $68 \%$ se declara internauta intensivo; es decir, han accedido a la Red en la última semana y representan el $35,7 \%$ de la población mayor de 15 años.

Internet se consolida como el servicio, utilizado en el hogar, que presenta una mejor relación precio-utilidad. En cuanto al acceso desde los hogares, la tasa de penetración (4) es incesante, situándose para el primer trimestre de 2007 en un 40\%; es decir 6,4 millones de hogares españoles están conectados a Internet, un 6,1\% más que en el mismo período del año anterior.

Adicionalmente, este mismo informe también arroja datos sorprendentes referidos a la enorme aceptación de la utilización de la eAdministración. Para el primer trimestre de 2007, el número de personas que contactaron con la Administración Pública a través de Internet ascendió a casi 7 millones (6.855.000), 570.000 individuos más que el mismo período del año anterior. Más de un $18 \%$ de la población de más de 15 años ya había contactado con la Administración Pública (General, Autonómica o Local) a través de Internet. Un $64,5 \%$ de los individuos que contactaron con la eAdministración en ese período consultó información referente a impuestos, un $44,7 \%$ lo hizo para encontrar información sobre becas y/o ayudas y un $34,8 \%$ para informarse sobre temas concernientes a empleo público.

También se advierte un cambio en las preferencias de los internautas respecto al lugar de conexión (5), imponiéndose el hogar frente al centro de trabajo o lugares que impliquen accesos más esporádicos (cibercafés, locutorios ...). Así, un $67 \%$ de los navegantes se conecta desde casa, frente al $42 \%$ que prefiere hacerlo desde el trabajo.

El acceso a Internet se ha democratizado extendiéndose a todos los públicos, con indepen- dencia de su situación económica, social o cultural. Esta popularización ha sido factible por la simplicidad (6) en la utilización de las nuevas tecnologías, lo que ha aumentado exponencialmente el número de usuarios con acceso a Internet; y porque en los últimos años la cantidad de servicios administrativos e informativos basados en la Web han proliferado.

Por último, reseñar que la expansión e importancia que las nuevas tecnologías están adquiriendo, en gran parte puede atribuirse a las nuevas técnicas para relacionarse socialmente y a la "empatía" de éstos con las TIC.

\section{Categorías de la discapacidad (7) y dispositivos de ayuda}

La supresión de barreras, tanto de infraestructuras, como sociales y culturales, constituye un tema de interés público y una preocupación que se ha visto plasmada (a veces por imperativo legal) en la obligación de adaptar infraestructuras y servicios para que éstos sean accesibles a toda la sociedad, consiguiendo así alcanzar uno de los derechos constitucionales más importantes, la igualdad de oportunidades.

Desde hace algún tiempo, el mundo de la informática también ha tenido que comenzar su particular adaptación para poder ser accesible a personas con discapacidades.

Conocer cuál es la situación de las personas discapacitadas ayuda, en gran medida, a entender la problemática a la que se enfrentan éstas en el momento de acceder a los sistemas de información. El conocimiento de aquellos casos de uso en los que la persona discapacitada encuentra dificultades facilitará la tarea de crear un contenido accesible, adquiriendo los conocimientos y las prácticas necesarios para que el proceso de adaptación de los contenidos a un formato accesible tenga un coste mínimo o prácticamente nulo. Además, la comprensión de las dificultades de adaptación a los sistemas de información también permite que los expertos en la técnica ideen fórmulas alternativas de acceso, como son los programas y dispositivos de asistencia que se adapten a la forma de trabajar de las personas discapacitadas (reduciendo las dificultades de acceso a Internet) en función del tipo de discapacidad de cada individuo.

El fenómeno de la discapacidad lleva aparejada una problemática que supera, con mucho, el tratamiento unitario de todas las posibles situaciones (ni siquiera de la mayoría de ellas. incluso ni clasificadas por tipologías de discapacidad); además, los diversos tipos de discapacidades no pueden considerarse, a priori, exclu- 
yentes entre sí, sino que en una misma persona pueden concurrir varias deficiencias (muy frecuente en personas de avanzada edad). Sin embargo, la agrupación en categorías sí favorece una aproximación más certera, no sólo al fenómeno en sí mismo considerado, sino también al esclarecimiento de las probables vías de mejora que reclama la situación actual de la discapacidad (entendida como la interacción entre el individuo y su entorno).

Se considera, pues, de gran trascendencia realizar una pequeña aproximación al concepto de discapacidad y sus tipos; describiendo aquellas discapacidades tanto de índole físico como técnico que padecen un número no desdeñable de usuarios, y que en la mayoría de los casos impiden su acceso a Internet, realizando una mención especial a la discapacidad visual, por ser ésta la que presenta mayor interés en esta investigación; además, por este mismo motivo se analizarán, en un mayor detalle, las aplicaciones informáticas destinadas a conseguir mayor accesibilidad de las páginas Web, con especial incidencia en aquéllas específicas para discapacitados visuales. En el apéndice, se incluyen en una tabla, a modo de resumen, las principales características de cada uno de estos tipos de deficiencias, clasificadas en función del órgano afectado; indicando además los programas o dispositivos aplicables para facilitar el acceso a los sistemas de información.

\section{Conclusiones}

La discapacidad, hoy en día, es un tema que preocupa a los poderes públicos y a la ciudadanía; por ello, está presente en todos los cuerpos legislativos. Sin embargo, en la actualidad, la sociedad sigue inmersa en los mismos problemas asociados a la discapacidad que hace tres décadas (salud, accesibilidad, educación, empleo ...). Esto se debe a que, pese a que se ha avanzado en la consecución de una mayor sensibilización por parte de los Gobiernos y de los agentes sociales en relación a la obligación que deben asumir para atender adecuadamente a sus ciudadanos con discapacidad, las acciones emprendidas (progresos legislativos, programas de distinta índole ...) no han sido capaces de acabar con la discriminación y exclusión social de este colectivo. Esto responde con total seguridad a la escasa participación de todos los actores implicados en el desarrollo de la sociedad. Es necesario acabar con las prácticas discriminatorias y eliminar barreras (socio-económicas, legales, psicológicas y referidas a la capacitación); para ello, es indispensable el acceso al mercado de trabajo, puesto que es el vehículo que conducirá a la integración plena de este colectivo en la sociedad. En este sentido, la accesibilidad electrónica se presenta como una herramienta altamente eficaz para la consecución de este objetivo.

Las telecomunicaciones y, en especial, Internet representan el más eficaz vehículo de transmisión e intercambio de información, de forma que su incorporación a la vida económica y social ofrece innumerables ventajas. Además, el acceso a las TIC crea oportunidades para todos, pues suponen una importante ayuda para que cualquier persona desarrolle sus posibilidades en las más diversas circunstancias a lo largo de la vida personal y profesional. Esta cualidad se acentúa en el caso de las personas con discapacidad, a las que permite, en mayor grado, formar parte e integrarse en el desarrollo de la sociedad. Sin embargo, también en este campo, las barreras sociales, de infraestructuras y de formatos inaccesibles obstaculizan esa participación. El colectivo de las personas con discapacidad se sitúa entre los sectores marginados de la sociedad, lo que hace que muchas de ellas no tengan acceso a las TIC e, incluso, aquéllas que sí lo tienen, a menudo no pueden utilizarlas con eficacia, pues el equipamiento adaptativo disponible no puede ajustarse a la velocidad de innovación de este sector. Estas dificultades de acceso y la carencia de un grado suficiente de conocimiento y familiaridad con tales tecnologías sitúan a las personas con discapacidad en una posición clara de desventaja frente al resto de la población. Pero, sobre todo, es indispensable el acceso al mercado de trabajo, puesto que es el vehículo que conducirá a la integración plena de este colectivo en la sociedad. La formación y el trabajo tienen una relación directa con el uso de las tecnologías de la información, siendo los dos ámbitos que más predisponen a Internet como herramienta de uso habitual.

El elevado porcentaje de población que existe con discapacidad en España, con especial incidencia en los discapacitados visuales, y el elevado porcentaje de usuarios de Internet, justifica la necesidad de impulsar las iniciativas para la accesibilidad y la no discriminación de estas personas. Además, los datos aquí vertidos ratifican que el número de personas destinatarias directas de un diseño Web accesible resulta lo suficientemente representativo como para preocuparse por satisfacer las necesidades de estos usuarios.

En este terreno, los poderes públicos pueden arbitrar diversas medidas impulsoras muy eficaces: una formación profesional ad hoc que permita su entrada en el empleo competitivo; un empleo protegido que palie las carencias de 
oferta en el competitivo; incentivos fiscales para las empresas colaboradoras y otras disposiciones legales; programas específicos, etc. A nivel internacional han aparecido normas que pretenden evitar la discriminación de estas personas en el ámbito de la Sociedad de la Información y que instan a los diferentes Gobiernos a adoptar la legislación necesaria para asegurarlo. No obstante, la mayoría de los actos legislativos emprendidos no tienen fuerza vinculante y en ningún caso generan obligaciones para los Estados miembros ni las instituciones europeas, ni derechos para sus destinatarios; aunque su contenido puede servir como punto de partida para la elaboración de normas con fuerza jurídica o para la realización de acciones positivas en este campo.

\section{Notas}

(1) La Ley de Servicios de la Sociedad de la Información y Comercio Electrónico (Ley 34/2002, de 11 de julio) establece, en su Disposición Adicional Quinta, con la denominación "Accesibilidad para las personas con discapacidad y de edad avanzada a la información proporcionada por medios electrónicos", la responsabilidad y obligación de las Administraciones Públicas, de los prestadores de servicios y los fabricantes de equipos y software para facilitar el acceso de las personas con discapacidad a los contenidos digitales. Obliga a ofrecer contenidos accesibles desde el 31 de diciembre de 2005 para todas las sedes de las Administraciones Públicas o de aquéllas cuyo diseño o mantenimiento sean financiados mediante fondos públicos.

(2) I Plan de Accesibilidad 2004-2012: Por un Nuevo Paradigma, el Diseño para Todos, hacia la Plena Igualdad de Oportunidades.

(3) Datos referidos al primer trimestre del 2007.

(4) Esta progresiva incorporación a Internet registrada en los últimos años lleva pareja una fidelización progresiva de los internautas, como así lo constata la antigüedad de acceso presentada por los mismos (el 63,8\% declararon haber usado la red por primera vez hace más de tres años).

(5) Los accesos a Internet no son excluyentes.

(6) Según el citado informe, Internet se consolida cada vez más como un medio fácil o muy fácil de utilizar (un $89 \%$ de los usuarios encuestados así lo valoraron); un medio que los usuarios utilizan cada vez más, puesto que éstos consideran que la Red cubre sus expectativas, considerándose satisfecho un $84 \%$ de ellos.

(7) En la Encuesta sobre Discapacidades, Deficiencias y Estado de Salud (INE, 1999), publicación de la cual se ha extraído gran parte de los datos reflejados en el presente trabajo, se consideran 36 discapacidades que se clasifican en 10 grupos principales: ver; oír; comunicarse; aprender, apli- car conocimientos y desarrollar tareas; desplazarse; utilizar brazos y manos; desplazarse fuera del hogar; cuidar de sí mismo; realizar las tareas del hogar; y relacionarse con otras personas.

(8) El código de comunicación Braille fue inventado en el siglo XIX por el profesor francés Louis Braille (Coupvray, departamento de Seine-et-Marne, cercanías de París, 1809/1852), ciego desde los tres años a causa de un accidente doméstico; actualmente, es casi el único método de lectura y escritura para ciegos y, sin lugar a dudas, el más universal.

\section{Referencias}

Corporate Social Responsibility. Labour standards for disabled employees: an employers' forum on disability briefing for CRS practitioners. [S.I.:s.n.], 2007. http:// www.csreurope.org/csrinfo/csrdisability/Disabledemploy ees/ (18-07-2007)

Elkouss, Eduardo (2006). La accesibilidad hacia la plena integración social del discapacitado en el entorno urbano y natural. Madrid: Instituto Juan de Herrera, 2006.

Fernández, Pedro (2005). La discapacidad y la integración laboral de personas discapacitadas: acercamiento a la realidad en la España Autonómica. // Cuadernos de Información Económica. 185 (marzo-abril, 2005).

Fundación de las Cajas de Ahorros: FUNCAS (2005). Transformación demográfica: raíces y consecuencias. // Papeles de Economía Española, 104 (2005). http:// www.funcas.ceca.es/Publicaciones/InformacionArticulos/ Publicaciones.asp?ld=1146 (18-07-2007).

Instituto Nacional de Estadística (España) (2005). Encuesta sobre Discapacidades, Deficiencias y Estado de Salud. Informe general. Madrid: INE, 2005. http://www.ine.es (02 -08-2007).

Instituto Nacional de Estadística (España) (2007). España en cifras: 2007. Madrid: INE, 2007. http://www.ine.es/ prodyser/pubweb/espcif/espcif07.htm (18-03-2007).

Laloma García, Miguel (2007). Empleo protegido en España: análisis de la normativa legal y logros alcanzados. Madrid: Cinca, 2007.

Ley $7 / 2007$, de 12 de abril, del Estatuto Básico del Empleado Público (B.O.E. núm. 89, de 13 de abril de 2007).

Ley 11/2007, de 22 de junio, de Acceso Electrónico de los Ciudadanos a los Servicios Públicos. (B.O.E. $\mathrm{n}^{\circ} 150$, de 23 de junio de 2007).

Ley 13/1982, de 7 de abril, de Integración Social de los Minusválidos (LISMI). (B.O.E. núm. 103, de 30 de abril de 1982).

Ley 20/2007, de 11 de julio, del Estatuto del Trabajo Autónomo (B.O.E. núm. 166, de 12 de julio de 2007).

Ley $34 / 2002$, de 11 de julio, de Servicios de la Sociedad de la Información y de Comercio Electrónico. (LSSICE). (B.O.E. $n^{\circ} 166$, de 12 de julio de 2002).

Ley 43/2006, de 29 de diciembre, para la Mejora del Crecimiento y del Empleo (B.O.E. núm. 312, de 30 de diciembre de 2006).

Ley $51 / 2003$, de 2 de diciembre, de Igualdad de Oportunidades, no Discriminación y Accesibilidad Universal de las Personas con Discapacidad. (B.O.E. $n^{\circ} 289$, de 3 de diciembre de 2003).

Ley 59/2003, de 19 de diciembre, de Firma Electrónica. (B.O.E. $n^{\circ} 304$, de 20 de diciembre de 2003).

Ley Orgánica $3 / 2007$, de 22 de marzo, para la Igualdad Efectiva de Mujeres y Hombres (B.O.E. núm. 71, de 23 de marzo de 2007). 
Lorenzo, Rafael de. El futuro de los discapacitados en el mundo: el empleo como factor determinante para la inclusión.//Revista del Ministerio de Trabajo y Asuntos Sociales, 50 (2004).73-89. http://www.mtas.es/publica/ revista/numeros/50/Est04.pdf (03-08-2007).

Maheux, Víctor M (2007). Las adaptaciones informáticas para discapacitados visuales. [S.I.:s.n], 2007. http://ww.e-santfeliu.org/online/pdf/adaptacions_invidene ts.pdf (12-01-2007).

Naciones Unidas (1982). Programa de Acción Mundial para las Personas con Discapacidad, adoptado por Resolución A/37/52 de la Asamblea General de las Naciones Unidas, de 3 de diciembre de 1982. [S.I.:s.n], 1982. http://www.geocities.com/leydiscaperu/programa_accion _mundial.htm (04-12-2006).

Nielsen, Jacob (2007). Beyond accessibility: treating people with disabilities as people. // Alertbox:Current Issues in Web Usability (2007). http://www.useit.com/alertbox/ 20011111.html (18-07-2007).

Observatorio de las Telecomunicaciones y la Sociedad de la Información (15a . Madrid. 2007). Panel de hogares: XV
Oleada del Observatorio de las Telecomunicaciones y la Sociedad de la Información: enero-marzo, 2007. Madrid:[S.n.], 2007. http:www.aui.es/IMG/pdf_Informe_Panel_Hogares_XV_ oleada.pdf (05-08-2007).

Oficina Internacional del Trabajo (Ginebra) (2007). La igualdad en el trabajo: afrontar los retos que se plantean. [Ginebra]: OIT, 2007. http://www.iol.org/declaration (0308-2007).

Organización Mundial de la Salud (Ginebra) (1980). Clasificación Internacional de Deficiencias, Discapacidades y Minusvalías: CIDDM. Ginebra: OMS, 1980. http:// www.who.int (12-01-2007).

Organización Mundial de la Salud (Ginebra) (2001). Clasificación Internacional del Funcionamiento, la Discapacidad y la Salud: CIFDS. Ginebra: OMS, 2001. http//www.who.int (12-01-2007).

Plan de Accesibilidad 2004-2012: Por un Nuevo Paradigma, el Diseño para Todos, hacia la Plena Igualdad de Oportunidades.

\section{Apéndice: El acceso a Internet según las principales discapacidades}

\begin{tabular}{|c|c|c|c|}
\hline & & Diagnóstico de su acceso a internet & Dispositivos o técnicas empleados para mitigar la barrera \\
\hline & 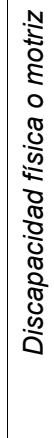 & $\begin{array}{l}\text { Estos usuarios no pueden utilizar } \\
\text { adecuadamente las extremidades } \\
\text { para interactuar con el ordenador a } \\
\text { través del teclado o del ratón, por lo } \\
\text { que suelen emplear dispositivos } \\
\text { alternativos, como los sistemas } \\
\text { basados en la voz u otros artilugios } \\
\text { como los licornios. }\end{array}$ & $\begin{array}{l}\text { Dispositivos alternativos: Tales como los sistemas basados en la voz u otros } \\
\text { mecanismos como el puntero electrónico o el licornio (dispositivo apuntador que } \\
\text { se coloca con un aro en la cabeza y por medio del cual el usuario puede tecle- } \\
\text { ar), teclados alternativos, joystick o ratones de tipo trackball. } \\
\text { Pantallas táctiles: Permiten la selección o activación del ordenador tocando la } \\
\text { pantalla. } \\
\text { Predicción de texto: Reducen el número necesario de pulsaciones de teclado. } \\
\text { Estos dispositivos permiten tener acceso rápidamente a las letras que comple- } \\
\text { tan las palabras, sin necesidad de escribirlas todas. } \\
\text { Sistemas de reconocimiento de voz. } \\
\text { Teclado en pantalla: Proporciona la imagen de un teclado en la pantalla para } \\
\text { que el usuario seleccione las teclas con un ratón, una pantalla táctil, un trackball, } \\
\text { un joystick, o un puntero electrónico. }\end{array}$ \\
\hline & 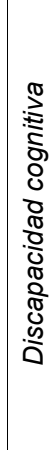 & $\begin{array}{l}\text { Gran parte de los usuarios afecta- } \\
\text { dos por estas deficiencias es perfec- } \\
\text { tamente capaz de aprender si la } \\
\text { información se les presenta de } \\
\text { forma apropiada; presentación } \\
\text { sencilla e impoluta. Durante el pro- } \\
\text { ceso de aprendizaje, puede ser } \\
\text { beneficioso tener una experiencia } \\
\text { multisensorial. Asimismo, una arqui- } \\
\text { tectura y maquetación adecuada de } \\
\text { la información, acompañada de la } \\
\text { reducción de distracciones visuales, } \\
\text { auditivas y del exceso de informa- } \\
\text { ción también puede ser beneficiosa. }\end{array}$ & $\begin{array}{l}\text { Comprensión de texto: Fortalecen las habilidades de lectura a través de activi- } \\
\text { dades, ejercicios o juegos. Ayudan a practicar el reconocimiento de las letras y a } \\
\text { fortalecer la comprensión de las palabras agregando gráficos, sonidos y anima- } \\
\text { ciones. } \\
\text { Predicción de texto. } \\
\text { Sintetizadores de voz. } \\
\text { Sistemas de reconocimiento de voz. }\end{array}$ \\
\hline & 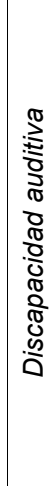 & $\begin{array}{l}\text { Los usuarios con algún grado de } \\
\text { pérdida de audición pueden oír un } \\
\text { cierto sonido, pero no distinguir las } \\
\text { palabras; para lograrlo, se puede } \\
\text { emplear un dispositivo que amplifi- } \\
\text { que el sonido. Sin embargo, los } \\
\text { sordos completos no pueden benefi- } \\
\text { ciarse de estos dispositivos. Estas } \\
\text { deficiencias pueden ser considera- } \\
\text { das menos limitadoras en el acceso } \\
\text { y uso de contenidos digitales, pues- } \\
\text { to que el canal sonoro en las interfa- } \\
\text { ces de los Sistemas de Información } \\
\text { no se utiliza con tanta frecuencia } \\
\text { como el canal visual. }\end{array}$ & $\begin{array}{l}\text { No existen productos específicos para este tipo de deficiencias, ya que pueden } \\
\text { recibir la información visualmente o bien, en función del grado de sordera, ajus- } \\
\text { tar el volumen de los sonidos a sus necesidades auditivas particulares. Además, } \\
\text { otra forma sencilla de solucionar el problema de la falta de audición puede ser } \\
\text { mediante periféricos que muestren avisos luminosos, o a través de una progra- } \\
\text { mación simple del ordenador para que los avisos luminosos se muestren por la } \\
\text { pantalla. } \\
\text { Educar/Reeducar el habla: Mediante el ordenador, periféricos comunes y soft- } \\
\text { ware ex profeso para estos propósitos, las personas con discapacidad auditiva } \\
\text { pueden conseguir o mejorar su educación oral. } \\
\text { Sistemas aumentativos y complementarios (SAAC) del habla: Los SAAC del } \\
\text { habla consisten principalmente en una serie de sistemas software encaminados } \\
\text { a aumentar y complementar la comunicación de las personas con deficiencia } \\
\text { auditiva. }\end{array}$ \\
\hline
\end{tabular}




\begin{tabular}{|c|c|c|}
\hline 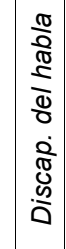 & $\begin{array}{l}\text { Para los usuarios con este tipo de } \\
\text { deficiencias, las representaciones } \\
\text { visuales complejas pueden dificultar } \\
\text { su accesibilidad, así como los textos } \\
\text { complejos o que presenten una } \\
\text { deficiente estructuración. }\end{array}$ & $\begin{array}{l}\text { Filtros de teclado. } \\
\text { Pantallas táctiles. } \\
\text { Revisión en pantalla: Convierten el texto que aparece en la pantalla en voz e } \\
\text { incluso destacan las palabras mientras se pronuncian; aportando la información } \\
\text { de modo tanto auditivo como visual. } \\
\text { Sintetizadores de voz. } \\
\text { Sistemas de reconocimiento de voz. }\end{array}$ \\
\hline 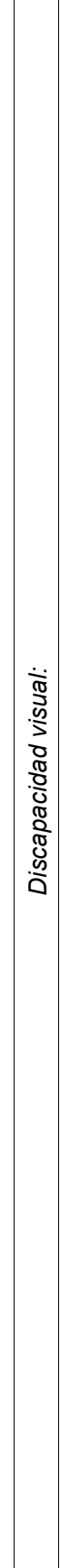 & $\begin{array}{l}\text { Los usuarios con esta discapacidad } \\
\text { tienen serias dificultades a la hora } \\
\text { de poder manejar con facilidad el } \\
\text { ordenador, pues éste está basado } \\
\text { sobre todo en estímulos visuales; } \\
\text { los principales problemas se en- } \\
\text { cuentran en la interacción con el } \\
\text { ordenador (los invidentes totales no } \\
\text { pueden utilizar el monitor y reciben } \\
\text { la información a través de sistemas } \\
\text { sensibles al sonido o al tacto). En el } \\
\text { caso de deficientes visuales, este } \\
\text { obstáculo puede sortearse con la } \\
\text { mera utilización del software ade- } \\
\text { cuado o parametrización de éste } \\
\text { (adaptación del software para que } \\
\text { muestre mayores contrastes, una } \\
\text { combinación de colores más eficaz, } \\
\text { un tamaño de letra superior o, inclu- } \\
\text { so, un zoom de la pantalla). En los } \\
\text { casos de ceguera deberíamos } \\
\text { adaptar los periféricos y utilizar } \\
\text { aquéllos, tanto de entrada como de } \\
\text { salida, específicos de este colectivo. }\end{array}$ & 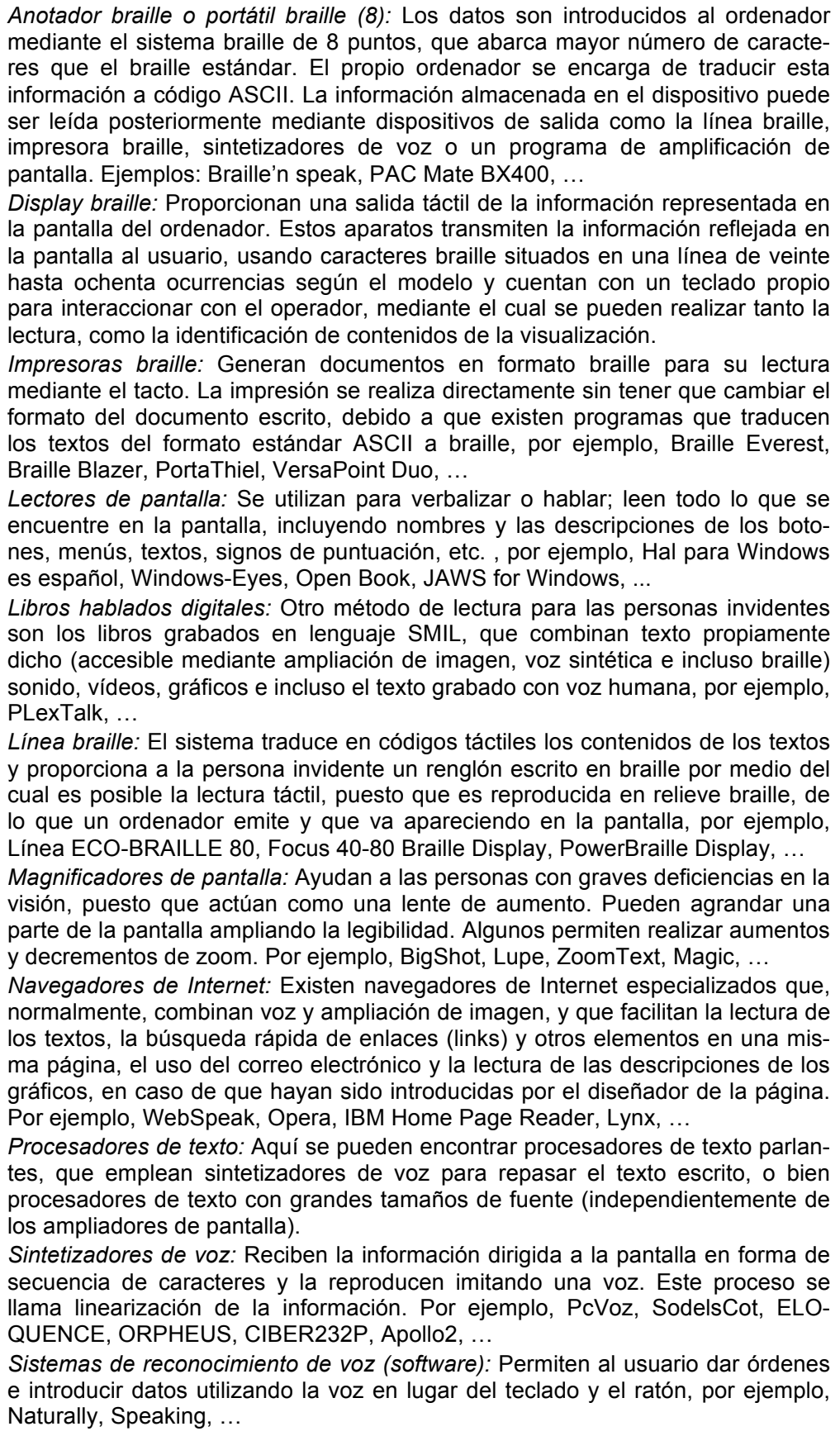 \\
\hline & $\begin{array}{l}\text { Determinados factores externos } \\
\text { como el acondicionamiento acústico, } \\
\text { lumínico o la realización simultánea } \\
\text { de distintas actividades pueden } \\
\text { dificultar también el acceso a la Red. } \\
\text { Por ello, también, deben tenerse en } \\
\text { cuenta estos condicionantes exter- } \\
\text { nos a la hora de diseñar sitios Web } \\
\text { accesibles. }\end{array}$ & $\begin{array}{l}\text { Están relacionadas con el equipo con el que un usuario accede a Internet, es } \\
\text { decir los dispositivos de acceso a la información. A continuación, se enumeran } \\
\text { algunos de éstos: navegadores desfasados o excesivamente nuevos, pero con } \\
\text { un uso poco extendido, o con opciones deshabilitadas; sistemas operativos } \\
\text { diferentes u obsoletos; dispositivos de visualización con un tamaño más peque- } \\
\text { ño que el estándar (PDA, teléfonos móviles, Palms, ...); pantalla en modo "sólo- } \\
\text { texto" o con baja resolución; tipo y calidad de la conexión (wireless, ...), etc. }\end{array}$ \\
\hline
\end{tabular}

